\title{
Extended gauge mediation in the NMSSM with displaced LHC signals
}

\author{
Marcin Badziak $^{1, \mathrm{a}}$, Nishita Desai ${ }^{2, \mathrm{~b}}$, Cyril Hugonie ${ }^{2, \mathrm{c}}$, Robert Ziegler ${ }^{3, \mathrm{~d}}$ \\ ${ }^{1}$ Faculty of Physics, Institute of Theoretical Physics, University of Warsaw, ul. Pasteura 5, 02-093 Warsaw, Poland \\ ${ }^{2}$ LUPM, UMR 5299, CNRS, Université de Montpellier, 34095 Montpellier, France \\ 3 Theoretical Physics Department, CERN, 1 Esplanade des Particules, 1211 Geneva 23, Switzerland
}

Received: 24 October 2018 / Accepted: 10 January 2019 / Published online: 28 January 2019

(C) The Author(s) 2019

\begin{abstract}
We analyze models of extended gauge Mediation in the context of the NMSSM, concentrating on supersymmetric spectra with light gluinos, low fine-tuning and decays of the lightest neutralino leading to displaced vertices. While the minimal scenario has rather heavy gluinos as a result of restrictions from the Higgs sector, we propose two new models in which the gluino can be as light as allowed by direct searches at the LHC, with a mass of about $1.7 \mathrm{TeV}$ and 2.0 TeV, respectively. Both models have a tuning of a few permille, and lead to an interesting phenomenology due to a light singlet sector. A singlet state at around $98 \mathrm{GeV}$ can account for the LEP excess, while the singlino has a mass of the order of $100 \mathrm{GeV}$ and decays to b-jets and the gravitino, with decay lengths of a few $\mathrm{cm}$.
\end{abstract}

\section{Introduction}

Gauge mediated supersymmetry breaking (GMSB) is one of the most elegant ways to explain the absence of sizable contributions to flavour violating processes in supersymmetric (SUSY) models. However, its simplest realizations are inconsistent with the measured Higgs mass of $125 \mathrm{GeV}$, unless one is willing to accept a very heavy (and thus unnatural) SUSY spectrum. In general there are two ways to make GMSB viable with sparticles light enough to be in the LHC reach. The first option to boost the Higgs mass is to maximize the loop corrections with sizable stop mixing, which requires direct Higgs-messenger couplings [1-21]. The second (less explored) option is to introduce additional tree-level contributions to the Higgs mass. Such corrections may originate

\footnotetext{
a e-mail: mbadziak@fuw.edu.pl

be-mail: nishita.desai@umontpellier.fr

c e-mail: cyril.hugonie@umontpellier.fr

de-mail: robert.ziegler@cern.ch
}

from the mixing of the Higgs with a lighter singlet [22], and can be realized in a simple model proposed by Delgado, Giudice and Slavich (DGS) [23] that combines the NMSSM and GMSB with direct singlet-messenger couplings. Indeed it has been shown in Ref. [24] that the most interesting region in parameter space features a light SUSY spectrum and a singlet around $90 \div 100 \mathrm{GeV}$, which gives a sizable tree-level contribution to the SM-like Higgs mass through mixing. Besides this "push-up" of the Higgs mass, the general framework of NMSSM+GMSB with a light singlet has other advantages. First, it provides a natural solution to the $\mu-B_{\mu}$ problem [23], as $\mu$ and $B_{\mu}$ are generated dynamically through the vacuum expectation value (VEV) of the singlet. Second, the Nextto-Lightest SUSY particle (NLSP) is typically the singlino, whose decay into the gravitino is suppressed, leading to novel displaced signatures at colliders [25].

In the present paper, we focus on GMSB + NMSSM models with direct matter-messenger couplings, with a special emphasis on displaced signatures. This is motivated by recent LHC results for heavy Higgs searches in the $\tau \tau$ channel [26], which has excluded the most interesting part of the parameter space of the DGS model. We investigate two simple extensions of the DGS model leading to a relatively light sparticle spectrum, which can be probed in the second run of the LHC, in particular associated with displaced decay signatures.

The rest of the article is organized as follows: In Sect. 2 we analyse the general features of SUSY models with light singlets. In Sect. 3 we review the DGS model and investigate the impact of recent LHC results on its parameter space. In Sects. 4 and 5 we introduce two new models with singletmessenger couplings, and summarize and conclude in Sect. 6. In an Appendix we provide complete expressions for soft terms in the respective models. 


\section{EWSB in the NMSSM with a light singlet}

Let us start with a brief discussion of Electroweak Symmetry Breaking (EWSB) in the $\mathbb{Z}_{3}$ invariant NMSSM, in which the $\mu$-term in the MSSM superpotential is replaced by the following singlet couplings

$W_{\mathrm{NMSSM}}=\lambda S H_{u} H_{d}+\frac{1}{3} \kappa S^{3}$,

and the NMSSM specific soft terms are given by

$$
\begin{aligned}
-\mathcal{L}_{\text {soft }} \supset m_{H_{u}}^{2}\left|H_{u}\right|^{2}+m_{H_{d}}^{2}\left|H_{d}\right|^{2}+m_{S}^{2}|S|^{2} \\
+\left(A_{\lambda} \lambda H_{u} H_{d} S+\frac{1}{3} \kappa A_{\kappa} S^{3}+\text { h.c. }\right) .
\end{aligned}
$$

In order to generate a sufficiently large VEV for the singlet $\langle S\rangle \equiv s$, the singlet soft mass $m_{S}^{2}$ must be negative or at least much smaller than $A_{\kappa}^{2}$. This statement can be quantified in the limit $s \gg v$, where $v$ is the electroweak scale, in which one finds

$s \approx \frac{A_{\kappa}}{\kappa} w, \quad w \equiv \frac{1+\sqrt{1-8 z}}{4}, \quad z \equiv \frac{m_{S}^{2}}{A_{\kappa}^{2}}$.

The approximate condition for proper EWSB in a global minimum of the potential reads

$z \lesssim \frac{1}{9} \Leftrightarrow w \gtrsim \frac{1}{3}$

In Minimal Gauge Mediation soft terms for the singlet sector do not arise at leading order which prevents successful EWSB. However, sufficiently large soft terms can be generated if there are direct couplings of the singlet to the messenger sector, as originally proposed in Ref. [27] and worked out in detail in Ref. [23]. It was further demonstrated in Ref. [24] that this model allows to realize the interesting "push-up" scenario where the SM-like Higgs mass gets a large positive contribution from mixing with a lighter singlet-like Higgs, thus allowing to lower the overall scale of the SUSY spectrum that drives the radiative corrections to the Higgs mass.

In the following we revisit this model (DGS) in the light of updated experimental constraints, and compare it to the phenomenology of two similar models that feature additional singlet-messenger couplings. We are particularly interested in SUSY spectra with experimental signatures that may be probed at the LHC using displaced vertices, as studied in Ref. [25].

Before discussing these models, we note that in their most interesting regions of the parameter space, where the SUSY spectrum can be relatively light thanks to the push-up effect of Higgs-singlet mixing, correct EWSB generically implies large values of $\tan \beta$. As a consequence, the most stringent constraints on these models often come from LHC searches for heavy MSSM-like Higgs bosons $H / A$ decaying to $\tau \tau$, because the cross-section for $H / A$ production grows with $\tan ^{2} \beta$. Since this constraint turns out so important, it is instructive to discuss the origin of large $\tan \beta$ in the push-up region of NMSSM models with gauge Mediation. Neglecting $m_{H_{d}}^{2}$, which is usually a good approximation in the models under consideration, the expression for $\tan \beta$ is relatively simple:

$\tan \beta \approx \frac{\lambda}{\kappa} \frac{A_{\kappa} w}{A_{\kappa} w-A_{\lambda}}, \quad \frac{\lambda^{2}}{\kappa^{2}} \approx \frac{\left(A_{\kappa} w-A_{\lambda}\right)^{2}-m_{H_{u}}^{2}}{A_{\kappa}^{2} w^{2}}$.

The soft parameters can in turn be related to the physical Higgs boson masses using the following approximate (treelevel) expressions for CP-odd Higgs masses:

$m_{a 1}^{2} \approx 3 A_{\kappa}^{2} w, \quad m_{a 2}^{2} \approx m_{H_{d}}^{2}-m_{H_{u}}^{2} \approx-m_{H_{u}}^{2}$,

the lightest (singlet-like) CP-even Higgs mass:

$m_{h_{1}}^{2} \approx A_{\kappa}^{2} w(4 w-1)-\delta m_{\text {mix }}^{2}$,

and the next-to-lightest (SM Higgs-like) CP-even Higgs mass

$m_{h_{2}}^{2} \approx \lambda^{2} v^{2} \sin ^{2} 2 \beta+M_{Z}^{2} \cos ^{2} 2 \beta+\delta m_{\text {mix }}^{2}$,

where $\delta m_{\text {mix }}^{2}$ is the contribution from singlet-doublet Higgs mixing and responsible for the "push-up" effect:

$\delta m_{\text {mix }}^{2} \approx 4 \lambda^{2} v^{2} \tan ^{2} \beta \frac{\left(A_{\kappa} w-A_{\lambda}\right)^{2}}{M_{Z}^{2}-A_{\kappa}^{2} w(4 w-1)}$,

where we anticipate $\tan \beta \gg 1$. We also note that for $\mu>$ 0 , which we always assume in this analysis ${ }^{1}, A_{\kappa}$ must be negative to avoid tachyons in the singlet sector. In the limit $w \gg 1$, corresponding to the case where EWSB is driven by a large negative $m_{S}^{2}$, one finds indeed large values of $\tan \beta$

$\tan \beta \approx \frac{\lambda}{\kappa} \approx \frac{\left|m_{H_{u}}\right|}{\left|A_{\kappa}\right| w} \approx \frac{2 m_{a 2}}{m_{h 1}} \gg 1$.

Using this relationship between $\tan \beta$ and $m_{a 2}$ at face value with $m_{h 1} \approx 100 \mathrm{GeV}$, the recent ATLAS constraints from heavy Higgs searches with $\tau \tau$ final states [26], implies a limit $\tan \beta \lesssim 25$ or equivalently $m_{a 2} \gtrsim 1.2 \mathrm{TeV}$, assuming no heavy Higgs decays to SUSY particles and neglecting threshold corrections to bottom quark Yukawa couplings. In typical GMSB models the value of $m_{a 2}$ is correlated to other sparticle masses, including squarks and gluino, so that

\footnotetext{
${ }^{1}$ Also $\mu<0$ leads to viable spectra, but we will not consider these scenarios in the following, since the gluino is always quite heavy with a mass above $3.2 \mathrm{TeV}$.
} 
a stronger bound on $m_{a 2}$ typically results in a stronger bound on coloured sparticles.

For smaller $w$ the value of $\tan \beta$ slightly decreases. In the limit $w \approx 1 / 3$ one finds

$\tan \beta \approx \frac{\left|m_{a 2}\right|}{m_{h 1}} \frac{1}{1-3 A_{\lambda} / A_{\kappa}} \approx \frac{\left|m_{a 2}\right|}{m_{h 1}} \frac{1}{1+A_{\lambda} / m_{h 1}} \gg 1$,

Neglecting the terms proportional to $A_{\lambda}, \tan \beta$ is smaller by a factor two with respect to the limit $w \gg 1$, so that the LHC constraints on the $a_{2}$ are expected to be relaxed. Taking into account non-zero $A_{\lambda}$, one can suppress (enhance) $\tan \beta$ when $A_{\lambda}$ is positive (negative). We also note that $A_{\lambda}$ may get positive contributions via RG running from a negative top trilinear term $A_{t}$.

Finally we provide an approximate expression for the $Z$ boson mass that is convenient to assess the fine-tuning. In the large $\tan \beta$ limit one has

$M_{Z}^{2} \approx-2 m_{H_{u}}^{2}-\frac{\lambda^{2}}{\kappa^{2}} \frac{A_{\kappa}^{2}}{4}(1-4 z+\sqrt{1-8 z})$,

with $z$ defined in Eq. (3). For the fine-tuning $\Delta$ we use the Barbieri-Giudice measure [28]

$\Delta \equiv \max _{i}\left\{\Delta_{\lambda_{i}}\right\}, \quad \Delta_{\lambda_{i}} \equiv \frac{\partial \log M_{Z}^{2}}{\partial \log \lambda_{i}^{2}}$,

where the maximum is taken over all UV parameters $\lambda_{i}$. For a thorough discussion of the tuning measure in extended GMSB models see e.g. Ref. [10].

We conclude this section with a brief comment of the potential tuning related to the "push-up" contribution to the SM-like Higgs mass, which can be relevant if $\lambda$ is sizable [29, 30]. In our case however, this source of tuning is absent, since $\lambda \ll 1$ (as we are going to see in the next sections), so that the contribution from mixing $\delta m_{\text {mix }}^{2}$ is small, (few $\left.\mathrm{GeV}\right)^{2}$, as compared to the total Higgs mass.

\section{The DGS model}

The field content of the DGS model [23] consists of the NMSSM fields (the MSSM fields plus a gauge singlet $S$ ), in addition to two copies of messengers in $\mathbf{5}+\overline{\mathbf{5}}$ representations of SU(5). The superpotential is given by the NMSSM (see Appendix for our conventions), the spurion-messenger couplings of ordinary gauge mediation and new singletmessenger couplings. Apart from the NMSSM part we have

$$
\begin{aligned}
W_{\text {DGS }}= & X \sum_{i=1}^{2}\left(\Phi_{u}^{(i)} \Phi_{d}^{(i)}+\Phi_{T}^{(i)} \Phi_{\bar{T}}^{(i)}\right) \\
& +S\left(\xi_{D} \Phi_{u}^{(1)} \Phi_{d}^{(2)}+\xi_{T} \Phi_{T}^{(1)} \Phi_{\bar{T}}^{(2)}\right)
\end{aligned}
$$

where $\Phi_{u}+\Phi_{d}$ and $\Phi_{T}+\Phi_{\bar{T}}$ denote the doublet and triplet components in $\mathbf{5}+\overline{\mathbf{5}}$, respectively, and $X$ denotes the SUSY breaking spurion that takes the $\operatorname{VEV}\langle X\rangle=M+F \theta^{2}$. This superpotential gives rise to soft SUSY breaking terms that can be found in the Appendix. These are determined by six parameters: the messenger scale $M$, the NMSSM couplings $\lambda$ and $\kappa$, the DGS couplings $\xi_{D}$ and $\xi_{T}$ and the effective scale of soft SUSY breaking terms $\tilde{m} \equiv 1 /\left(16 \pi^{2}\right) F / M$. One of these parameters (following DGS we choose $\kappa$ ) can be eliminated by requiring correct EWSB. We also impose a unification condition for $\xi_{D}$ and $\xi_{T}$ that allows to eliminate one additional parameter,

$\xi_{D}\left(M_{\mathrm{GUT}}\right)=\xi_{T}\left(M_{\mathrm{GUT}}\right)=\xi$.

In the following analysis we will always assume this relation, but we have checked that the more general case of independent $\xi_{D}$ and $\xi_{T}$ leads to similar phenomenology.

For heavy singlet-like scalars, all sparticles must be very heavy in order to satisfy the Higgs mass constraint in this model, and fall outside the discovery reach of the LHC [23]. It was found in Ref. [24] that a significantly lighter SUSY spectrum is possible in the presence of a light singlet that pushes up the Higgs mass via Higgs-singlet mixing. Having the light singlet mass fixed around $90 \div 100 \mathrm{GeV}$ (where the constraints on the Higgs-singlet mixing from LEP [31] are weakest) implies $\xi \sim 10^{-2}$, while the Higgs-singlet mixing maximizing the push-up effect on the Higgs mass requires $\lambda \sim 10^{-2}$. For such small values of the couplings one has $m_{S}^{2} \approx-16 \tilde{m}^{2} \xi_{T}^{2} g_{3}^{2}$, so that the condition in Eq. (4) for correct EWSB is always fulfilled. As a result, the DGS model with a light singlet has $w \gg 1$, so that $\tan \beta$ is approximately given by Eq. (10). An interesting prediction of the DGS model with a light singlet is a singlino NLSP with mass about $m_{\tilde{N}_{1}} \approx$ $100 \mathrm{GeV}$ that decays to gravitino LSP and $a_{1}$ or $h_{1}$, which typically decay further to $b \bar{b}$. An estimate for the decay length up to $\mathcal{O}(1)$ factors is given by

$c \tau_{\tilde{N}_{1}} \approx 2.5 \mathrm{~cm}\left(\frac{100 \mathrm{GeV}}{M_{\tilde{N}_{1}}}\right)^{5}\left(\frac{M}{10^{6} \mathrm{GeV}}\right)^{2}\left(\frac{\tilde{m}}{\mathrm{TeV}}\right)^{2}$.

Therefore the singlino is long-lived and results in displaced vertices within the LHC detectors for sufficiently low values of the messenger scale $M \lesssim 10^{7} \mathrm{GeV}$, corresponding to an NLSP decay length $c \tau_{\tilde{N}_{1}} \lesssim \mathcal{O}(10) \mathrm{m}$. However, in order to see such a long-lived singlino at the LHC, it must have 
been produced from the decay of a heavy SUSY particle like gluino or squark, since the direct production of a singlino is negligible due its small couplings. Therefore displaced signatures require a sufficiently light colored spectrum, which might be in conflict with existing LHC searches.

In order to assess this issue, we have updated the analysis of the DGS model in Ref. [24] using the latest LHC constraints. For this analysis we have used the public code NMSSMTOols [32,33], which computes the SUSY and Higgs spectrum and checks the latest LHC constraints in the Higgs sector (LHC constraints on SUSY sector will be dealt with later with CheckMATE2). We have performed large MCMC scans of the parameter space of the DGS model and sorted the results in a $2 \mathrm{D}$ histogram in the plane of the gluino mass vs. the decay length of the singlino NLSP. In each bin of this histogram, we have kept the point in parameter space minimising the fine-tuning as defined in Eq. (11). In Fig. 1 we present a map of fine-tuning in the plane of the gluino mass and the decay length of the singlino NLSP. We see that for NLSP decay lengths corresponding to a displaced vertex, the gluino mass is pushed far beyond $3 \mathrm{TeV}$. Thus, the DGS model does not predict any displaced signatures that could be observed at the LHC. We also note that even for larger NLSP decay length, for which the NLSP is stable from the LHC perspective, the gluino must be heavy and may be beyond the LHC discovery reach. Numerically, we find a lower bound on the gluino mass of $1.8 \mathrm{TeV}$, and a lower bound on the tuning of $\Delta \geq 600$.

These negative conclusions can be traced back to recent heavy Higgs searches in the $\tau \tau$ channel [26], which exclude previously viable points with light gluinos. This happens because successful EWSB in the DGS model with a light singlet requires rather large values of $\tan \beta \gtrsim 30$, which pushes the heavy MSSM-like Higgs bosons to values above $1.3 \mathrm{TeV}$ [26], and thus requires larger SUSY scales.

Therefore, in order to have a light sparticle spectrum that can be tested at the LHC, a model of NMSSM + GMSB is desirable that can provide sufficiently heavy MSSM-like Higgs bosons. In the DGS model the gluino mass is correlated with $m_{a 2}$ since the scale of both parameters is set by $\tilde{m}$. In order to avoid stringent lower bounds on the gluino mass, one should look for a model in which the correlation between gluino mass and $m_{a 2}$ is broken by new contributions to the UV soft masses and/or $w \approx 1 / 3$ (for which $\tan \beta$ is generically smaller so the LHC constraints on $m_{a 2}$ are weaker). In the following sections we present two models that satisfy these requirements and therefore simultaneously allow both for a gluino much lighter than in the DGS model and sufficiently small singlino decay lengths to have displaced vertices at the $\mathrm{LHC}$.

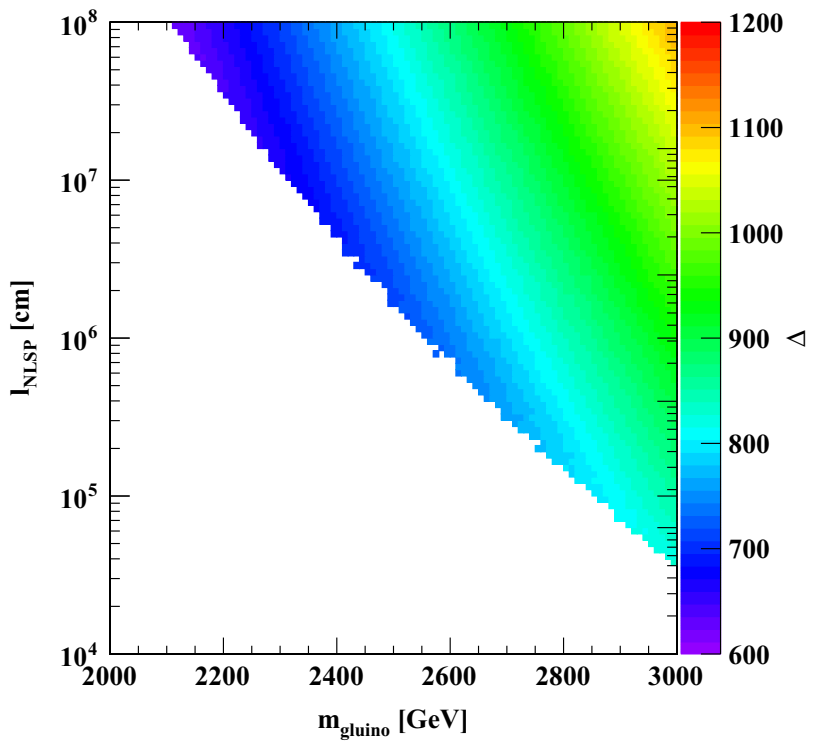

Fig. 1 Gluino mass vs. NLSP decay length $c \tau_{\tilde{N}_{1}}$ in the DGS model after applying all current LHC constraints. The colour map indicates the values of the fine-tuning measure $\Delta$

\section{The U model}

In this new model, we take one copy of messengers and add all possible linear couplings of the messenger $\Phi_{u}$ :

$$
\begin{aligned}
W_{\mathrm{U}}= & X\left(\Phi_{u} \Phi_{d}+\Phi_{T} \Phi_{\bar{T}}\right) \\
& +\lambda_{t} Q_{3} U_{3} \Phi_{u}+\lambda_{S_{d}} S \Phi_{u} H_{d} .
\end{aligned}
$$

This gives rise to soft SUSY breaking terms that can be found in the Appendix, and are now determined by six parameters: $\tilde{m}, M, \lambda, \kappa, \lambda_{t}$ and $\lambda_{S_{d}}$. Note that one might impose the condition $\lambda_{S_{d}} y_{t}=\lambda_{t} \lambda$ that would result from explicit messenger-Higgs mixing [1]. However, we found that for small values of $\lambda \lesssim 10^{-2}$ (as required to avoid experimental constraints on the Higgs-singlet mixing), $\lambda_{S_{d}}$ would be too small to allow for $m_{h_{1}}$ to be in the preferred window between about 90 and $100 \mathrm{GeV}$ in order to to significantly enhance the Higgs mass. Thus, in the following we assume that $\lambda_{S_{d}}$ and $\lambda_{t}$ are independent parameters, so that there is one additional parameter as compared to the DGS model.

In Fig. 2 we present a map of fine-tuning in the plane of the gluino mass and the decay length of the singlino NLSP, obtained analogously to Fig. 1. One can clearly distinguish two different regions here, in the upper part the "DGS-like" region, that is characterised by a large NLSP decay length $c \tau_{\tilde{N}_{1}} \gtrsim 100 \mathrm{~cm}$, small fine-tuning $\Delta \lesssim 10^{3}$, a light singlet spectrum $\left(m_{a_{1}} \approx 30 \div 40 \mathrm{GeV}, m_{h 1} \approx 90 \mathrm{GeV}\right.$, $m_{\tilde{S}} \approx 100 \mathrm{GeV}$ ) and an essentially constant input value $\lambda_{S_{d}} \sim 10^{-2}$. Representative for the DGS-like region are the benchmark points P1 and P2 in Table 1. A closer look reveals that this region actually falls into two sub-regions, 


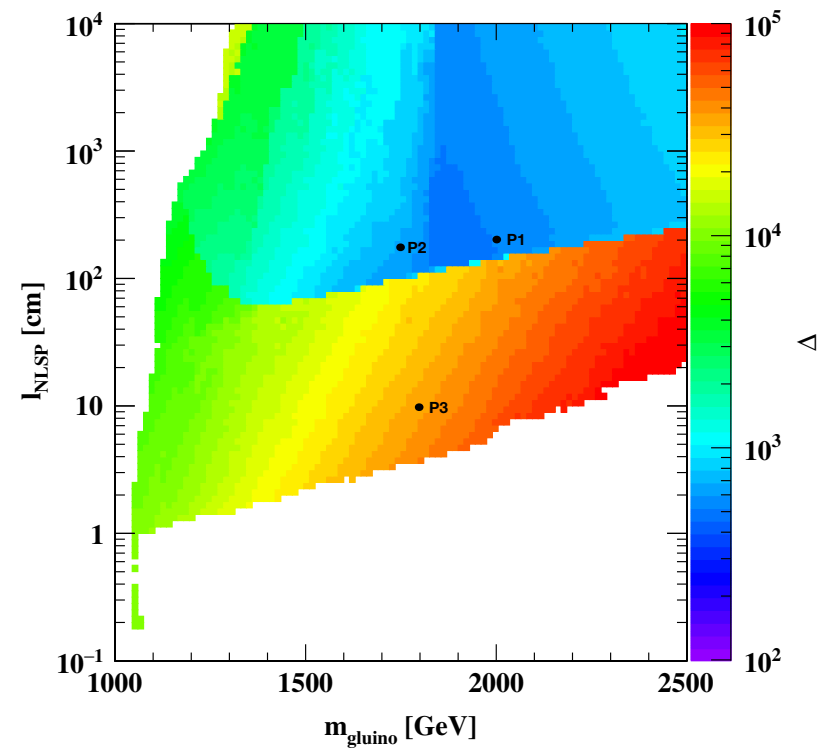

Fig. 2 The same as in Fig. 1 but for the U model and without applying constraints from LHC sparticle searches. Also shown are the benchmark points P1-P3 of Table 1

which can be separated by the value of the gluino mass as visible in Fig. 2. For gluino masses above $1.8 \mathrm{TeV}$, the value of $\left|\lambda_{t}\right|$ is essentially constant, $\sim 0.3$, while it increases up to $\sim 0.7$ towards lighter masses. Moreover, these parts are distinguished by the value of $\tan \beta$, which is about 10 in the left part and about 20 in the heavy gluino part. The latter is represented by P1 while P2 exemplifies the light gluino part of the DGS region.

The other region with smaller NLSP decay lengths $c \tau_{\tilde{N}_{1}} \lesssim$ $100 \mathrm{~cm}$ is represented by benchmark P3 in Table 1. This region features much larger tuning $\Delta \gg 10^{3}$, essentially constant $\tan \beta \approx 10$, a heavier singlet spectrum $\left(m_{a 1}, m_{\tilde{N}_{1}} \gtrsim\right.$ $200 \mathrm{GeV})$, large constant values of $\left|\lambda_{t}\right| \approx 0.7$ and $\lambda_{S_{d}} \approx 0.2$ and a potentially lighter gluino mass compared to the DGSlike region. From Fig. 2 one can see that a singlino decay length $\mathcal{O}(1) \mathrm{cm}$ is possible for a gluino as light as about $1 \mathrm{TeV}$, without being in conflict with Higgs sector constraints. Nevertheless, such a light gluino might be already excluded by direct searches at the LHC.

We have therefore taken into account the LHC limits from direct SUSY searches with CheckMATE2 [34]. To briefly summarise the workflow, CheckMATE2 uses Pythia8 [35] to generate all accessible $2 \rightarrow 2$ processes followed by a detector simulation with Delphes 3 [36]. Variables and cuts used in experimental analyses are then implemented as closely as possible to "recast" the analysis, and the expected number of signal events that pass the cuts are validated against published benchmarks and cut flows. This validated analysis can then be used to test New Physics models against published upper limits. In the presence of multiple signal regions that may potentially be sensitive to model predictions, CheckMATE2 selects only the most sensitive region with respect to the expected background.

We summarize the results in Fig. 3 in the same parameter space as in Fig. 2, where red (green) points are excluded (allowed) by current LHC constraints. This plot shows that direct LHC searches still allow for a gluino as light as about 1.7 TeV, for essentially any NLSP decay length. The bound is significantly weaker than in typical simplified models presented by the experimental collaborations. The main reason for the relaxed constraints is that the wino is lighter than the left-handed sleptons of the first two generations, and therefore dominantly decays to the lightest stau (which has some non-negligible left-handed component), resulting in $\tau$ 's instead of leptons in the final state. This feature strongly relaxes the mass limits both for direct production of winos, as well as production of gluinos decaying predominantly to winos. We found that the most constraining searches for this model are the ATLAS searches with two same-sign leptons or tri-leptons [37], and jets and missing energy [38].

Let us now discuss how such a light gluino in the U model can be compatible with the constraints from the SM-like Higgs mass and experimental searches for heavy Higgses. First of all, the points with a light gluino feature large $\lambda_{t}$, which implies large $A_{t}$, so that the Higgs mass is enhanced not only by the push-up effect but also by the loop contribution from stop mixing. However, we recall that in the DGS model the lower bound on the SUSY scale does not arise from the Higgs mass constraint, but from direct searches for heavy MSSM-like Higgs bosons. In the U model instead, the pseudoscalar Higgs mass $m_{a_{2}}$ is enhanced by large $\lambda_{t}$, as a result of the contribution to the soft Higgs mass parameter $m_{H_{u}}^{2} \sim-9 y_{t}^{2} \lambda_{t}^{2} \tilde{m}^{2}$, cf. (A.14). Moreover, larger values of $\lambda_{t}$ are correlated with smaller $\tan \beta$, as shown in Fig. 4. This is because larger $\lambda_{t}$ results in smaller $w$, which follows from

$z=\frac{m_{S}^{2}}{A_{\kappa}^{2}} \approx \frac{1}{6 \lambda_{S_{d}}^{2}}\left(\lambda_{t}^{2}-g_{2}^{2}\right)$

and Eq. (3). For $\lambda_{t} \gtrsim 0.7, w$ approaches $1 / 3$ for which $\tan \beta$ is twice as small as in the limit $w \gg 1$, as explained ${ }^{2}$ in Sect. 2. Therefore, the combined effect of increased $m_{a 2}$ and decreased $\tan \beta$ renders the LHC searches for heavy Higgs bosons with $\tau \tau$ final states essentially insensitive to the $\mathrm{U}$ model with large $\lambda_{t}$ and light gluinos.

We however notice that the points with light gluinos are more fine-tuned than DGS-like solutions with smaller $\lambda_{t}$ and gluinos above $1.8 \mathrm{TeV}$. This is because the tuning associated with $\lambda_{t}$ dominates the total tuning in almost all points of

\footnotetext{
${ }^{2}$ For large $\lambda_{t}, \tan \beta$ is suppressed even more due to the RG effect of $A_{t}$ which makes $A_{\lambda}$ positive at the EW scale and cannot be neglected in Eq. (11). This effect is partially compensated by the fact that $\lambda_{t}$ increases $m_{a 2}$ that enters in the numerator of the formula for $\tan \beta$ in Eq. (11).
} 
Table 1 Benchmarks consistent with all experimental constraints, including LHC direct search limits. P1-P3 are points in the U model, while P4-P6 belong to the DGSU model. All points have reduced SMlike Higgs signal strengths of about 0.84 , corresponding to a Higgs- singlet mixing angle of $\cos \theta \approx 0.92$, while the signal strengths of the singlet-like state are about 0.16 . All soft masses are at the SUSY scale. We note that Higgs decays with $a_{1}$ in final states are strongly suppressed due to small $\lambda$

\begin{tabular}{|c|c|c|c|c|c|c|}
\hline & $\mathrm{P} 1$ & $\mathrm{P} 2$ & $\mathrm{P} 3$ & $\mathrm{P} 4$ & P5 & P6 \\
\hline$\tilde{m}[\mathrm{TeV}]$ & 1.7 & 1.5 & 1.5 & 0.87 & 1.0 & 1.0 \\
\hline$M[\mathrm{GeV}]$ & $2.8 \times 10^{6}$ & $3.1 \times 10^{6}$ & $2.5 \times 10^{6}$ & $5.6 \times 10^{6}$ & $5.1 \times 10^{6}$ & $1.6 \times 10^{6}$ \\
\hline$\lambda$ & $4.6 \times 10^{-3}$ & $4.4 \times 10^{-3}$ & $1.1 \times 10^{-3}$ & $4.9 \times 10^{-3}$ & $5.4 \times 10^{-3}$ & $2.5 \times 10^{-3}$ \\
\hline$\kappa$ & $1.4 \times 10^{-4}$ & $1.2 \times 10^{-4}$ & $4.3 \times 10^{-5}$ & $1.5 \times 10^{-4}$ & $2.1 \times 10^{-4}$ & $6.5 \times 10^{-5}$ \\
\hline$\lambda_{t}$ & -0.33 & -0.48 & -0.73 & -0.64 & -0.38 & 0.76 \\
\hline$\lambda S_{d}$ & 0.022 & 0.028 & 0.17 & - & - & - \\
\hline$\xi$ & - & - & - & 0.012 & 0.010 & 0.010 \\
\hline $\tan \beta$ & 18 & 11 & 10 & 9.1 & 17 & 8.7 \\
\hline$m_{\tilde{g}}[\mathrm{TeV}]$ & 2.0 & 1.7 & 1.8 & 2.0 & 2.3 & 2.2 \\
\hline$m_{\tilde{d}_{R}}[\mathrm{TeV}]$ & 2.6 & 2.2 & 2.3 & 2.0 & 2.4 & 2.3 \\
\hline$m_{\tilde{t}_{1}}[\mathrm{TeV}]$ & 2.1 & 1.7 & 2.2 & 1.8 & 2.0 & 2.2 \\
\hline$m_{\tilde{N}_{1}}[\mathrm{GeV}]$ & 95 & 96 & 200 & 96 & 97 & 106 \\
\hline$m_{\tilde{N}_{2}}[\mathrm{GeV}]$ & 370 & 320 & 330 & 380 & 440 & 430 \\
\hline$m_{a_{1}}[\mathrm{GeV}]$ & 26 & 32 & 290 & 26 & 24 & 26 \\
\hline$m_{a_{2}}[\mathrm{TeV}]$ & 1.7 & 1.9 & 2.7 & 1.6 & 1.4 & 2.1 \\
\hline$m_{h_{1}}[\mathrm{GeV}]$ & 89 & 89 & 110 & 89 & 91 & 101 \\
\hline$m_{\mu L}[\mathrm{GeV}]$ & 960 & 830 & 820 & 710 & 840 & 790 \\
\hline$m_{\mu R}[\mathrm{GeV}]$ & 480 & 430 & 520 & 390 & 420 & 450 \\
\hline$m_{\chi_{1}^{ \pm}}[\mathrm{GeV}]$ & 720 & 620 & 640 & 720 & 840 & 830 \\
\hline$m_{H_{u}}^{2}\left[\mathrm{TeV}^{2}\right]$ & -2.2 & -2.8 & -6.8 & -2.2 & -1.5 & -3.9 \\
\hline$m_{H_{d}}^{2}\left[\mathrm{TeV}^{2}\right]$ & 0.75 & 0.62 & 0.59 & 0.47 & 0.58 & 0.58 \\
\hline$m_{S}^{2}\left[\mathrm{GeV}^{2}\right]$ & $-4.3 \times 10^{3}$ & $-4.3 \times 10^{3}$ & $7.8 \times 10^{3}$ & $-4.3 \times 10^{3}$ & $-4.5 \times 10^{3}$ & $-5.3 \times 10^{3}$ \\
\hline$A_{\lambda}[\mathrm{GeV}]$ & 64 & 130 & 180 & 150 & 52 & 210 \\
\hline$A_{\kappa}[\mathrm{GeV}]$ & -4.9 & -7.2 & -280 & -4.8 & -3.9 & -4.3 \\
\hline$c \tau_{\tilde{N}_{1}}[\mathrm{~cm}]$ & 200 & 200 & 10 & 200 & 200 & 12 \\
\hline$\Delta$ & 530 & 680 & 37000 & 440 & 310 & 720 \\
\hline
\end{tabular}

the $\mathrm{U}$ model. Using the approximate expression for $M_{Z}^{2}$ in Eq. (12), one can easily show that the $\lambda_{t}$ contribution to the tuning $\Delta_{\lambda_{t}}$ is approximately given by

$\Delta_{\lambda_{t}} \approx 6 \lambda_{t}^{2} \frac{\tilde{m}^{2}}{M_{Z}^{2}}\left(3 y_{t}^{2}+\frac{\lambda^{2}}{\kappa^{2}} \lambda_{S_{d}}^{2}\right)$

where the first term is the contribution to $m_{H_{u}}^{2}$, and the second is the contribution to the effective $\mu$-term.

While $\lambda^{2} / \kappa^{2} \sim 10^{3}$ is essentially constant in both regions, the smallness of $\lambda_{S_{d}} \sim 10^{-2}$ in the DGS-like region implies that the second term in Eq. (19) is small, and the tuning is essentially determined by $\lambda_{t}^{2} \tilde{m}^{2}$, with a minimal value of $\Delta \approx$ 450. Instead the other region features large $\lambda_{S_{d}} \sim 0.2$, which together with large $\lambda_{t}$ leads to a significant enhancement of the tuning with respect to the DGS-like region, by about a factor 100. This estimate explains the large increase in the tuning when crossing between the two different regions in Fig. 2. It also accounts for the approximately constant tuning over the two DGS-like sub-regions, where a decrease in the gluino mass (i.e. $\tilde{m}$ ) is compensated by an increase in $\lambda_{t}$, such that the total tuning remains essentially constant, up to $\mathcal{O}(1)$ factors.

We conclude this section with a discussion of the three benchmark points P1-P3 in Table 1, which represent sample spectra of the U model with a light SUSY spectrum compatible with all LHC constraints, with a singlino decay length roughly below two meters. The point P1 features a tuning of $\Delta \approx 500$, with a gluino mass around $2 \mathrm{TeV}$ and NLSP singlino decay length about $2 \mathrm{~m}$. This point represents the DGS-like region in the upper right corner with large $\tan \beta$. The point $\mathrm{P} 2$ has larger $\lambda_{t} \approx 0.5$, which allows for a lighter gluino, although the tuning slightly increases as discussed above. The larger $\lambda_{t}$ implies smaller $\tan \beta \approx 11$, 


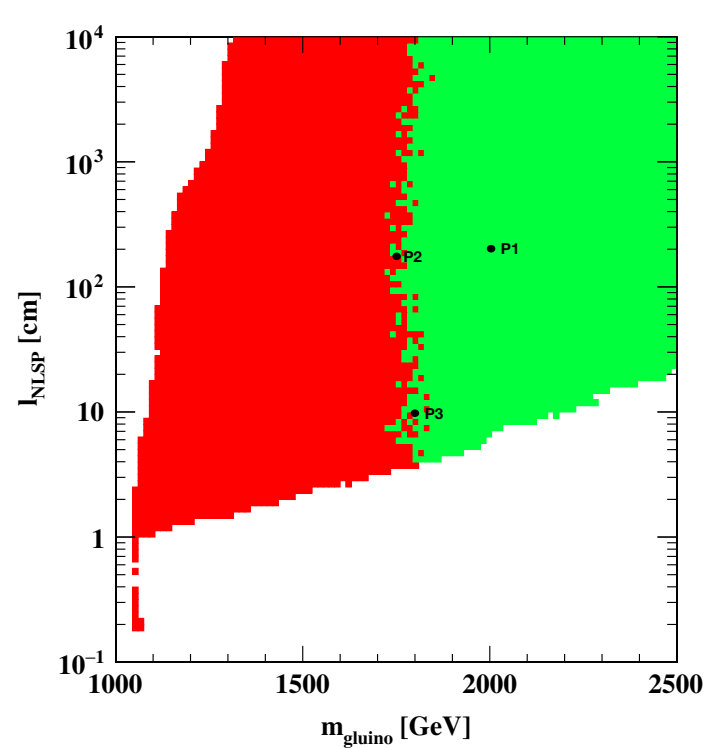

Fig. 3 Exclusion limits in the U model obtained with CheckMATE2. Red (green) points are excluded (allowed) by direct SUSY searches at the LHC. Also shown are the benchmark points P1-P3 of Table 1

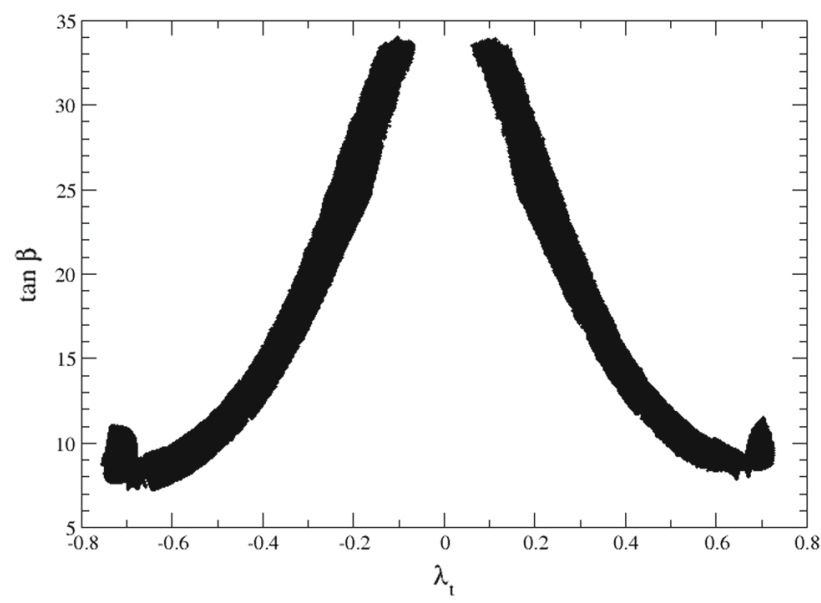

Fig. 4 Correlation of $\tan \beta$ and $\lambda_{t}$ for points with $m_{\tilde{g}}<2.5 \mathrm{TeV}$ and $c \tau_{\mathrm{R}_{1}}<10^{4} \mathrm{~cm}$ displayed in Figs. 2 and 3

while the light singlet spectrum is quite similar to P1, with a NLSP singlino decay length that is still relatively large, $c \tau_{\tilde{N}_{1}} \approx 1.9 \mathrm{~m}$. For both P1 and P2, the singlino NLSP mainly decays to the gravitino and the lightest pseudoscalar $a_{1}$ (with mass $\sim 30 \mathrm{GeV}$ ) which in turns decays promptly to $b \bar{b}$. Characteristic for these benchmarks is a light Higgs state $h_{1}$ with mass around $90 \mathrm{GeV}$, which is mainly a SM singlet with a small doublet admixture corresponding to a mixing angle $\cos ^{2} \theta \approx 0.84$. This state might account for the $2 \sigma$ excess observed at LEP $[31,39]$ in the $b \bar{b}$ channel, and could also explain the tentative hint for a light Higgs decaying into diphotons at CMS [40], although the signal strength of the light singlet state in the diphoton channel is about a factor of three smaller than the current central value of the CMS excess.

On the other hand, NLSP decay lengths below $1 \mathrm{~m}$ can only be obtained for the price of increased fine-tuning, in the region where $\lambda_{t} \approx 0.7$ is constant. This is exemplified by the point $\mathrm{P} 3$, which features $c \tau_{\tilde{N}_{1}} \approx 10 \mathrm{~cm}$ and a tuning of at least $\Delta \approx 10^{4}$. These points have a heavier Higgs spectrum, and in particular the lightest pseudoscalar is now heavier than 250 $\mathrm{GeV}$, while the NLSP singlino is above $200 \mathrm{GeV}$. In contrast to $\mathrm{P} 1$ and $\mathrm{P} 2$, here the singlino mainly decays to gravitino and $h_{1}$ (with mass $\sim 110 \mathrm{GeV}$ ), since $a_{1}$ is too heavy.

\section{The DGSU model}

Motivated by the previous analysis, we finally consider a model that combines the virtues of the DGS and the U model, thus allowing for light gluinos, small singlino decay lengths and low tuning. In this scenario, dubbed the "DGSU model", we take two messenger copies (i.e. $N=2$ ) and introduce the following couplings:

$$
\begin{aligned}
W_{\mathrm{DGSU}}= & S\left(\xi_{D} \Phi_{u}^{(1)} \Phi_{d}^{(2)}+\xi_{T} \Phi_{T}^{(1)} \Phi_{\bar{T}}^{(2)}\right) \\
& +\lambda_{t} Q_{3} U_{3} \Phi_{u}^{(2)}+\lambda_{S_{d}} S \Phi_{u}^{(2)} H_{d}
\end{aligned}
$$

We impose the DGS unification condition at the GUT scale

$\xi_{D}\left(M_{\mathrm{GUT}}\right)=\xi_{T}\left(M_{\mathrm{GUT}}\right)=\xi$

In contrast to the $\mathrm{U}$ model we $\mathrm{can}^{3}$ now impose the Higgsmessenger mixing condition (for simplicity at the messenger scale)

$\lambda_{S_{d}}(M) y_{t}(M)=\lambda_{t}(M) \lambda(M)$.

The superpotential couplings lead to additional contributions that can be found in the Appendix, and are given in terms of the six independent parameters $\tilde{m}, M, \lambda, \kappa, \xi$ and $\lambda_{t}$ (the same number as in the U model).

In Fig. 5 we present a map of fine-tuning in the plane of the gluino mass and the decay length of the singlino NLSP, obtained analogously to Figs. 1 and 2. However, comparing to the exclusion limits from direct LHC searches shown in Fig. 6, we see that they are stronger than in the $U$ model and the allowed points can have gluino masses only slightly below $2 \mathrm{TeV}$. The reason for this is that in the DGSU model there are two messengers (in contrast to one in the U model). Since the minimal gauge mediation contribution to gaugino masses is proportional to the number of messengers $N$ while

\footnotetext{
3 This is because the singlet scalar mass is now set by $\xi \sim 10^{-2}$ as in the DGS model, instead of $\lambda_{S_{d}}$ as in the U model.
} 


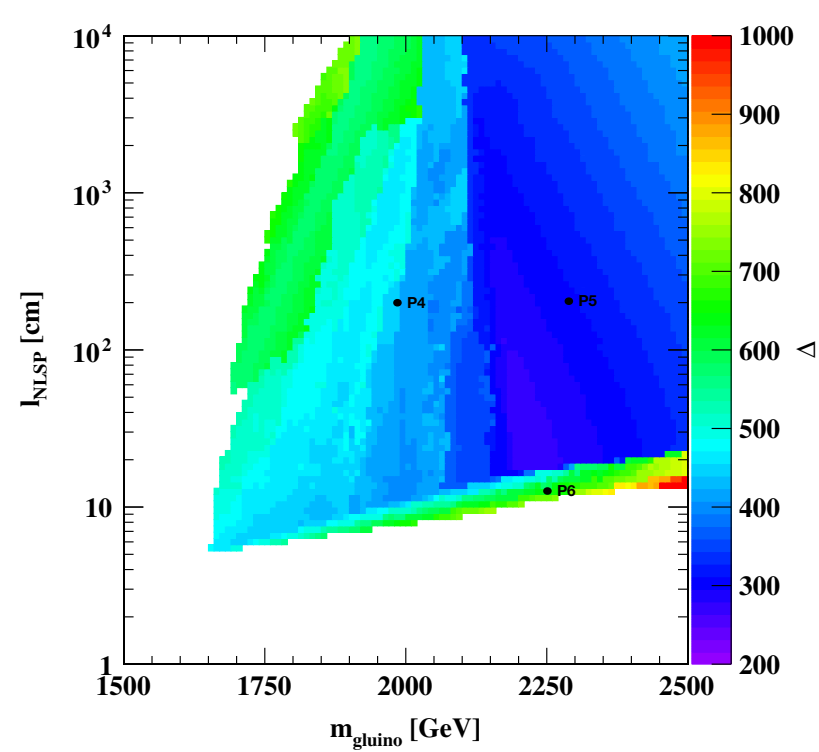

Fig. 5 The same as in Figs. 1 and 2, but for the DGSU model and without applying constraints from LHC sparticle searches. Also shown are the benchmark P4-P6 of Table 1

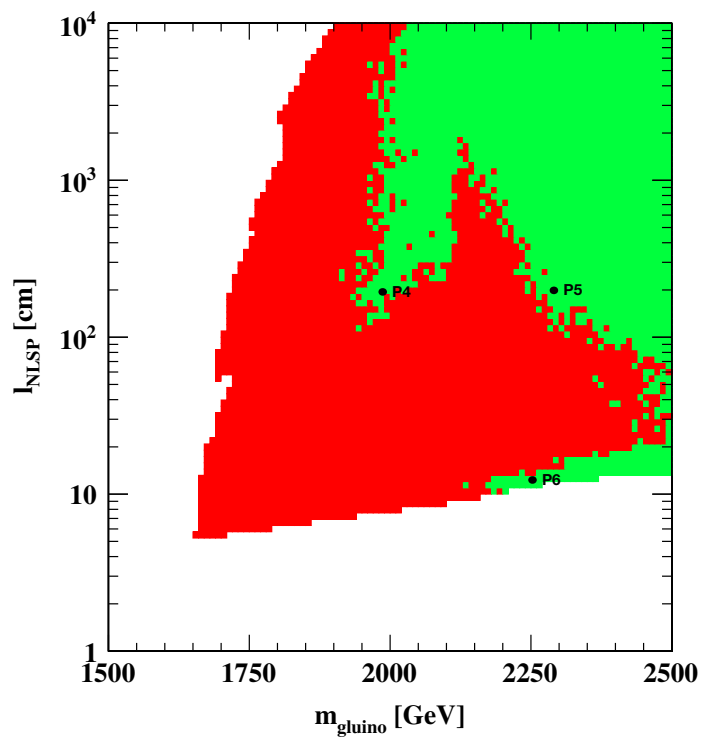

Fig. 6 Exclusion limits in the DGSU model obtained with CheckMATE2. Red (green) points are excluded (allowed) by direct SUSY searches at the LHC. Also shown are the benchmark points P4P6 of Table 1

the corresponding contribution to sfermion masses scales as $\sqrt{N}$, cf. (A.3), this makes sfermions lighter for a given gaugino masses. As a result, the squarks of the first two generations are comparable to the gluino mass and their production cross-section is non-negligible. For the same reason left-handed sleptons of the first two generations can now be lighter than wino, which results in more leptons in the final state (instead of $\tau$ 's as in the U model). The latter feature of the DGSU model also explains why the constraints for larger NLSP decay lengths are weaker, since the larger decay length is obtained for larger messenger scale, which in turn results in heavier sleptons (as compared to gauginos) due to their stronger renormalization by electroweak gauginos. Nevertheless, a gluino mass of $2 \mathrm{TeV}$ is still viable for a decay length $\mathcal{O}(1) \mathrm{m}$, while decay lengths roughly between 20 and $100 \mathrm{~cm}$ implies a limit for the gluino mass of almost $2.5 \mathrm{TeV}$. We should also emphasize that this limit is particularly strong not only due to gluino/squark production, but also due to direct production of sleptons and winos, whose mass is correlated with the gluino mass. Therefore also direct searches for direct electroweak production set important constraints [41], besides analyses using jets and missing energy.

Note that for decay lengths of $\mathcal{O}(10) \mathrm{cm}$, there is a small strip of allowed points (represented by benchmark point P6), which however correspond to larger tuning, cf. Fig. 5. This is a consequence of large values of $\left|\lambda_{t}\right|$, which also explains the relaxed LHC constraints. For large values of $\left|\lambda_{t}\right|$, there is a large positive contribution to stop masses at the messenger scale, cf. (A.14), which leads to heavier stop masses at low scales, so that gluino decays to stops are kinematically forbidden.

We notice that the DGSU model shares many features with the DGS model, or rather the DGS-like region of the U model. In particular, all points (represented by benchmarks P4-P6 in Table 1) have a well-defined singlet sector with a singlet-like scalar $h_{1}$ around $90-100 \mathrm{GeV}$, a singlet-like pseudoscalar $a_{1}$ around $20-30 \mathrm{GeV}$ and a singlino NLSP between $90-100$ $\mathrm{GeV}$. The singlino NLSP decay length can be rather short, of the order of $\mathcal{O}(10) \mathrm{cm}$, and it decays mainly to gravitino and $a_{1}$, which decays promptly to $b \bar{b}$. The input parameters are essentially constant throughout the whole region, with $\lambda \sim 10^{-3}$ and $\xi \sim 10^{-2}$. As in the DGS-like region of the $\mathrm{U}$ model one can further distinguish two sub-regions, which are characterised by different values of $\left|\lambda_{t}\right|$ (that is essentially constant $\sim 0.4$ for gluino masses above $\sim 2.2 \mathrm{TeV}$, and below this value starts increasing towards lighter gluino masses, up to $\sim 0.8$ ) and $\tan \beta$, which is correlated with $\lambda_{t}$ similar as in Fig. 4 . These two parameters essentially control the mass of the MSSM-like pseudoscalar $a_{2}$ and the tuning. The mass of the former grows for larger $\lambda_{t}$ (a feature inherited from the $\mathrm{U}$ model), and thus is no longer correlated with the gluino mass so that $H / A \rightarrow \tau \tau$ searches do not constrain this model. Moreover, large values of $\lambda_{t}$ lead to a large contribution to the Higgs mass from stop mixing, which implies that the overall SUSY scale can be lowered, and gluinos and stops can be quite light. The DGSU model is thus a perfect realization of the pNMSSM scenario analysed in Ref. [25], and motivates the combined searches using displaced and prompt signatures advocated in that article.

Finally, the DGSU model is also much less fine-tuned than the points with light gluinos in the $\mathrm{U}$ model. This can be again understood from the fact that $\lambda_{t}$ (which is sizable) 
dominates the total tuning, and contributes as in Eq. (19) with $\lambda_{S_{d}}$ replaced by $\xi$. Similar to the DGS-like region of the $\mathrm{U}$ model, this contribution is small, so that the tuning is controlled by $\lambda_{t}^{2} \tilde{m}^{2}$, with a minimal ${ }^{4}$ value of $\Delta \approx 300$, which is reached for intermediate values for the gluino mass around $2.3 \mathrm{TeV}$, where the product of $\lambda_{t}$ and $m_{\tilde{g}}$ is minimal.

\section{Summary and conclusions}

In this article we have analyzed models of extended Gauge Mediation in the context of the NMSSM. The simplest scenario (DGS) requires rather heavy gluinos, which are constrained not by direct SUSY searches but rather a two-fold restriction coming from the Higgs sector. On the one hand, the mass of the SM-like Higgs requires sizable loop corrections, on the other hand direct LHC Higgs searches in the $\tau \tau$ channel set stringent lower limits on the mass of the heavy Higgses, which in turn is tied to the SUSY scale. We have proposed two new models with singlet/Higgs-messenger couplings (the U model and the DGSU model), which ease these constraints and thus allow for gluinos as light as allowed by direct searches. First, the presence of a direct coupling of stops to the messenger fields generate sizable $A_{t}$ at the messenger scale, which in turn allows for much lighter stops (and thus also other sparticles) consistent with the measured SM-like Higgs mass. Second, the same coupling also generates new contributions to the soft Higgs mass, which allows to both increase the MSSM-like Higgs doublet mass and reduce $\tan \beta$, such that the LHC searches for heavy Higgs are satisfied without raising the overall SUSY scale. To identify the lower bound on the gluino mass in these models, we have recasted the existing LHC searches and found that gluinos can be as light as about $1.7 \mathrm{TeV}$ (U model) and $2.0 \mathrm{TeV}$ (DGSU model). The tuning in both models is rather low, and can be as small as $2 \%$ o (U model) and 3\% (DGSU model). The phenomenology is quite different in the two models: in the $U$ model there are essentially two distinct regions, one with a DGS-like spectrum featuring a light singlet sector, large singlino decay lengths roughly above a meter (but small enough to see a displaced vertex in the LHC detectors) and low fine-tuning. The other region is characterized by a heavy singlet sector, less displaced singlino decays with $c \tau_{\tilde{N}_{1}} \approx 1 \div 10 \mathrm{~cm}$ and large tuning. The DGSU model combines the most interesting phenomenological features of both regions: light gluinos in the reach of LHC, a light singlet sector with a $98 \mathrm{GeV}$ state (that might account for the LEP excess and improves the fit to the CMS data hinting at a new light state decaying to $\gamma \gamma$ ), and displaced singlino decays into $b \bar{b}+$ MET with decay lengths as small as a few $\mathrm{cm}$. In

\footnotetext{
4 This is a factor of a few smaller than the minimal tuning found for a broad class of extended GMSB models in the context of the MSSM [10].
}

Table 1 we have collected benchmark points representative for the relevant parameter regions and the two models.

Acknowledgements The authors acknowledge the support of FranceGrilles and the OCEVU Labex (ANR-11-LABX-0060) for providing computing resources on the French National Grid Infrastructure, and support from the French research project Defi InFIniti - AAP 2017. MB and RZ thank the Galileo Galilei Institute for Theoretical Physics and INFN for hospitality and partial support during the completion of this work. MB has been partially supported by the National Science Centre, Poland, under the research grant no. 2017/26/D/ST2/00225. N.D. was supported by the OCEVU Labex (ANR-11-LABX-0060) and the A*MIDEX project (ANR-11-IDEX0001-02) funded by the "Investissements d'Avenir" French government program managed by the ANR.

Data Availability Statement This manuscript has no associated data or the data will not be deposited. [Authors comment: The datasets generated and analysed for the current study are available from the corresponding author on reasonable request.]

Open Access This article is distributed under the terms of the Creative Commons Attribution 4.0 International License (http://creativecomm ons.org/licenses/by/4.0/), which permits unrestricted use, distribution, and reproduction in any medium, provided you give appropriate credit to the original author(s) and the source, provide a link to the Creative Commons license, and indicate if changes were made.

Funded by $\mathrm{SCOAP}^{3}$.

\section{Appendix A: models}

In this Appendix we provide further details about the models discussed in the main text, including the full superpotential, its motivation by symmetries, and the complete list of soft terms (obtained using the results of Ref. [10]).

\section{NMSSM + gauge mediation}

In order to fix notation, we define the NMSSM and minimal gauge mediation by the superpotential

$$
\begin{aligned}
W= & X \sum_{i=1}^{N}\left(\Phi_{u}^{(i)} \Phi_{d}^{(i)}+\Phi_{T}^{(i)} \Phi_{\bar{T}}^{(i)}\right)+\lambda S H_{u} H_{d}+\frac{\kappa}{3} S^{3} \\
& +Q^{T} y_{U} U H_{u}+Q^{T} y_{D} D H_{d}+L^{T} y_{E} E H_{d},
\end{aligned}
$$

where $X$ denotes the SUSY breaking spurion that takes the vev $\langle X\rangle=M+F \theta^{2}, N$ is the number of messengers in complete $\mathbf{5}+\overline{\mathbf{5}}$ representations of SU(5), and $\Phi_{u}+\Phi_{d}$ and $\Phi_{T}+\Phi_{\bar{T}}$ denote the doublet and triplet components in $\mathbf{5}+\overline{\mathbf{5}}$, respectively. The spurion vev will induce soft masses and A-terms defined as

$$
\begin{aligned}
-\mathcal{L}= & \tilde{q}_{L}^{T} \tilde{m}_{Q}^{2} \tilde{q}_{L}^{*}+\tilde{u}_{R}^{T} \tilde{m}_{U}^{2} \tilde{u}_{R}^{*}+\tilde{d}_{R}^{T} \tilde{m}_{D}^{2} \tilde{d}_{R}^{*}+\tilde{l}_{L}^{T} \tilde{m}_{L}^{2} \tilde{l}_{L}^{*} \\
& +\tilde{e}_{R}^{T} \tilde{m}_{E}^{2} \tilde{e}_{R}^{*}+\tilde{m}_{H_{u}}^{2}\left|H_{u}\right|^{2}+\tilde{m}_{H_{d}}^{2}\left|H_{d}\right|^{2}+\tilde{m}_{S}^{2}|S|^{2} \\
& +A_{U} \tilde{q}_{L}^{T} y_{U} \tilde{u}_{R}^{*} H_{u}+A_{D} \tilde{q}_{L}^{T} y_{D} \tilde{d}_{R}^{*} H_{d}+A_{E} \tilde{l}_{L}^{T} y_{E} \tilde{e}_{R}^{*} H_{d} \\
& +\lambda A_{\lambda} S H_{u} H_{d}+\kappa \frac{A_{\kappa}}{3} S^{3} .
\end{aligned}
$$


Table 2 Quadratic Casimirs of MSSM fields

\begin{tabular}{llllllll}
\hline & $Q$ & $U$ & $D$ & $L$ & $E$ & $H_{u}$ & $H_{d}$ \\
\hline $\mathrm{SU}(3)$ & $4 / 3$ & $4 / 3$ & $4 / 3$ & 0 & 0 & 0 & 0 \\
$\mathrm{SU}(2)$ & $3 / 4$ & 0 & 0 & $3 / 4$ & 0 & $3 / 4$ & $3 / 4$ \\
$\mathrm{U}(1)$ & $1 / 60$ & $4 / 15$ & $1 / 15$ & $3 / 20$ & $3 / 5$ & $3 / 20$ & $3 / 20$ \\
\hline
\end{tabular}

Table 3 Charge assignments in the DGS model

\begin{tabular}{lllllll}
\hline & $X$ & $H_{u}$ & $H_{d}$ & $S$ & $\Phi_{\mathbf{5}}^{1}, \Phi_{\overline{\mathbf{5}}}^{2}$ & $\Phi_{\overline{\mathbf{5}}}^{1}, \Phi_{\mathbf{5}}^{2}$ \\
\hline$U(1)_{Z}$ & 1 & -1 & 1 & 0 & 0 & -1 \\
$Z_{3}$ & 0 & 0 & 2 & 1 & 1 & 2 \\
\hline
\end{tabular}

Without introducing additional couplings, the A-terms vanish at the messenger scale and gaugino masses and sfermion masses are given by the usual minimal gauge mediation expressions:

$M_{i}=N g_{i}^{2} \tilde{m}, \quad \tilde{m}_{f}^{2}=2 N \sum_{i=1}^{3} C_{i}(f) g_{i}^{4} \tilde{m}^{2}$,

where $\tilde{m} \equiv 1 /\left(16 \pi^{2}\right) F / M$ and $C_{i}(f)$ is the quadratic Casimir of the representation of the field $f$ under SU(3) $\times$ $\mathrm{SU}(2) \times \mathrm{U}(1)$, for completeness given in Table 2 .

In particular the soft mass of the singlet vanishes at the messenger scale, $\tilde{m}_{S}^{2}=0$. Together with the vanishing Aterms, this makes it difficult to trigger EWSB in minimal gauge mediation and motivates the introduction of additional interactions among messengers and NMSSM fields.

\section{DGS model}

The following singlet-messenger couplings are added to the superpotential of Eq. (A.1):

$W_{\mathrm{DGS}}=S\left(\xi_{D} \Phi_{u}^{(1)} \Phi_{d}^{(2)}+\xi_{T} \Phi_{T}^{(1)} \Phi_{\bar{T}}^{(2)}\right)$

Notice that two copies of messengers are introduced (i.e. $N=$ 2 ) in order to avoid that $S$ has the same quantum numbers of $X$, which would lead to tadpoles terms that destabilize the hierarchy. The superpotential in Eq. (A.1) and Eq. (A.4) is the most general one allowed by a $U(1)_{Z} \times Z_{3}$ symmetry with quantum numbers as in Table 3.

Note that one can impose a unification condition for $\xi_{D}$ and $\xi_{T}$ that allows to eliminate one parameter,

$\xi_{D}\left(M_{\mathrm{GUT}}\right)=\xi_{T}\left(M_{\mathrm{GUT}}\right)=\xi$.

The new couplings leads to additional contributions to soft terms, on top of the soft terms from minimal GM in Eq. (A.3).
Table 4 Charge assignments in the $\mathrm{U}$ model

\begin{tabular}{lllllll}
\hline & $X$ & $H_{u}$ & $H_{d}$ & $S$ & $\Phi_{\mathbf{5}}$ & $\Phi_{\overline{\mathbf{5}}}$ \\
\hline$U(1)_{Z}$ & 1 & 0 & 0 & 0 & 0 & -1 \\
$Z_{3}$ & 0 & 1 & 1 & 1 & 1 & 2 \\
\hline
\end{tabular}

Now A-terms for the singlet are generated at one loop:

$A_{\lambda}=-\left(2 \xi_{D}^{2}+3 \xi_{T}^{2}\right) \tilde{m}$

$A_{\kappa}=-3\left(2 \xi_{D}^{2}+3 \xi_{T}^{2}\right) \tilde{m}$.

A soft mass for the singlet arises at one loop with an additional $F / M^{2}$ suppression that renders this contribution relevant only for very low messenger scales:

$\left.\tilde{m}_{S}^{2}\right|_{1-\text { loop }}=-16 \pi^{2} \tilde{m}^{2} \frac{F^{2}}{M^{4}} \frac{h\left(F / M^{2}\right)}{3}\left(2 \xi_{D}^{2}+3 \xi_{T}^{2}\right)$,

with the loop function

$h(x) \equiv \frac{3}{x^{3}} \log \frac{1-x}{1+x}-\frac{6}{x^{4}} \log \left(1-x^{2}\right)=1+\frac{4}{5} x^{2}+\mathcal{O}\left(x^{4}\right)$.

Unsuppressed soft masses in the Higgs sector are generated at two loops:

$$
\begin{aligned}
\tilde{m}_{S}^{2}= & -\tilde{m}^{2}\left[\xi_{D}^{2}\left(6 / 5 g_{1}^{2}+6 g_{2}^{2}\right)+\xi_{T}^{2}\left(4 / 5 g_{1}^{2}+16 g_{3}^{2}\right)\right] \\
& -\tilde{m}^{2}\left[4 \kappa^{2}\left(2 \xi_{D}^{2}+3 \xi_{T}^{2}\right)\right] \\
& +\tilde{m}^{2}\left[8 \xi_{D}^{4}+15 \xi_{T}^{4}+12 \xi_{D}^{2} \xi_{T}^{2}\right], \\
\Delta \tilde{m}_{H_{u}}^{2}= & \Delta \tilde{m}_{H_{d}}^{2}=-\tilde{m}^{2} \lambda^{2}\left(2 \xi_{D}^{2}+3 \xi_{T}^{2}\right) .
\end{aligned}
$$

\section{U model}

In this model, we take just one messenger copy, $N=1$, and introduce the following couplings with $\Phi_{u}$ :

$W_{\mathrm{U}}=\lambda_{t} Q_{3} U_{3} \Phi_{u}+\lambda_{S_{d}} S \Phi_{u} H_{d}$

The superpotential in Eq. (A.1) and Eq. (A.15) is the most general one allowed by a $U(1)_{Z} \times Z_{3}$ symmetry with quantum numbers given in Table 4 , where $H_{u}$ is defined as that field that does not couple to $X$.

One can also impose the condition

$\lambda_{S_{d}} y_{t}=\lambda_{t} \lambda$ 
that would result from a explicit messenger-Higgs mixing [1].

These couplings leads to additional contributions to soft terms, on top of the soft terms from minimal GM in Eq. (A.3). For one-loop A-terms one finds:

$$
\begin{aligned}
\left(A_{U}\right)_{33} & =-3 \lambda_{t}^{2} \tilde{m}, \\
\left(A_{D}\right)_{33} & =-\left(\lambda_{t}^{2}+\lambda_{S_{d}}^{2}\right) \tilde{m}, \\
\left(A_{E}\right)_{33} & =-\lambda_{S_{d}}^{2} \tilde{m} \\
A_{\lambda} & =-3 \lambda_{S_{d}}^{2} \tilde{m} \\
A_{\kappa} & =-6 \lambda_{S_{d}}^{2} \tilde{m} .
\end{aligned}
$$

Also masses for $Q_{3}, U_{3}, S$ and $H_{d}$ are generated at one loop, but with an additional suppression by $F / M^{2}$ :

$$
\begin{aligned}
\left.\Delta \tilde{m}_{Q_{3}}^{2}\right|_{1-\text { loop }} & =-16 \pi^{2} \tilde{m}^{2} \frac{F^{2}}{M^{4}} \frac{h\left(F / M^{2}\right)}{6} \lambda_{t}^{2}, \\
\left.\Delta \tilde{m}_{U_{3}}^{2}\right|_{1-\text { loop }} & =-16 \pi^{2} \tilde{m}^{2} \frac{F^{2}}{M^{4}} \frac{h\left(F / M^{2}\right)}{3} \lambda_{t}^{2}, \\
\left.\tilde{m}_{S}^{2}\right|_{1-\text { loop }} & =-16 \pi^{2} \tilde{m}^{2} \frac{F^{2}}{M^{4}} \frac{h\left(F / M^{2}\right)}{3} \lambda_{S_{d}}^{2}, \\
\left.\Delta \tilde{m}_{H_{d}}^{2}\right|_{1-\text { loop }} & =-16 \pi^{2} \tilde{m}^{2} \frac{F^{2}}{M^{4}} \frac{h\left(F / M^{2}\right)}{6} \lambda_{S_{d}}^{2},
\end{aligned}
$$

where the loop function $h(x)$ is defined in Eq. (A.8). The two-loop soft masses are:

$$
\begin{aligned}
\Delta m_{H_{u}}^{2}= & -3\left(3 y_{t}^{2} \lambda_{t}^{2}+\lambda_{S_{d}}^{2} \lambda^{2}\right) \tilde{m}^{2}, \\
\Delta m_{H_{d}}^{2}= & 6 \lambda \lambda_{S_{d}} \lambda_{t} y_{t} \tilde{m}^{2}-\lambda_{S_{d}}^{2}\left(3 / 5 g_{1}^{2}+3 g_{2}^{2}\right) \tilde{m}^{2} \\
& -3 y_{b}^{2} \lambda_{t}^{2} \tilde{m}^{2}+\lambda_{S_{d}}^{2}\left(2 \kappa^{2}+4 \lambda_{S_{d}}^{2}+2 \lambda^{2}+3 \lambda_{t}^{2}\right) \tilde{m}^{2}, \\
\tilde{m}_{S}^{2}= & \lambda_{S_{d}}^{2}\left(-6 / 5 g_{1}^{2}-6 g_{2}^{2}-8 \kappa^{2}+4 \lambda^{2}+8 \lambda_{S_{d}}^{2}\right) \tilde{m}^{2} \\
& +\left[\lambda_{S_{d}}^{2}\left(6 \lambda_{t}^{2}+6 y_{b}^{2}+2 y_{\tau}^{2}\right)+12 \lambda \lambda_{S_{d}} \lambda_{t} y_{t}\right] \tilde{m}^{2}, \\
\Delta \tilde{m}_{Q_{3}}^{2}= & \lambda_{t}^{2}\left(-13 / 15 g_{1}^{2}-3 g_{2}^{2}-16 / 3 g_{3}^{2}+6 y_{t}^{2}\right) \tilde{m}^{2} \\
& +\left(6 \lambda_{t}^{4}+\lambda S_{S_{d}}^{2}\left(\lambda_{t}^{2}-y_{b}^{2}\right)+2 \lambda \lambda_{S_{d}} \lambda_{t} y_{t}\right) \tilde{m}^{2}, \\
\Delta \tilde{m}_{U_{3}}^{2}= & \lambda_{t}^{2}\left(-26 / 15 g_{1}^{2}-6 g_{2}^{2}-32 / 3 g_{3}^{2}+12 y_{t}^{2}\right) \tilde{m}^{2} \\
& +\left(12 \lambda_{t}^{4}+2 y_{b}^{2} \lambda_{t}^{2}+2 \lambda_{S_{d}}^{2} \lambda_{t}^{2}+4 \lambda \lambda_{S_{d}} y_{t} \lambda_{t}\right) \tilde{m}^{2}, \\
\Delta \tilde{m}_{D_{3}}^{2}= & -2\left(y_{b}^{2} \lambda_{t}^{2}+\lambda_{S_{d}}^{2} y_{b}^{2}\right) \tilde{m}^{2}, \\
\Delta \tilde{m}_{L_{3}}^{2}= & -\lambda_{S_{d}}^{2} y_{\tau}^{2} \tilde{m}^{2}, \\
\Delta \tilde{m}_{E_{3}}^{2}= & -2 \lambda_{S_{d}}^{2} y_{\tau}^{2} \tilde{m}^{2} .
\end{aligned}
$$

Table 5 Charge assignment in the DGSU model

\begin{tabular}{lllllll}
\hline & $X$ & $H_{u}$ & $H_{d}$ & $S$ & $\Phi_{\mathbf{5}}^{1}, \Phi_{\overline{\mathbf{5}}}^{2}$ & $\Phi_{\overline{\mathbf{5}}}, \Phi_{\mathbf{5}}^{2}$ \\
\hline$U(1)_{Z}$ & 1 & -1 & 1 & 0 & 0 & -1 \\
$Z_{3}$ & 0 & 2 & 0 & 1 & 1 & 2 \\
\hline
\end{tabular}

\section{DGSU model}

In this model, we take two messenger copies, $N=2$, and introduce the following couplings:

$$
\begin{aligned}
W_{\mathrm{DGSU}}= & S\left(\xi_{D} \Phi_{u}^{(1)} \Phi_{d}^{(2)}+\xi_{T} \Phi_{T}^{(1)} \Phi_{\bar{T}}^{(2)}\right) \\
& +\lambda_{t} Q_{3} U_{3} \Phi_{u}^{(2)}+\lambda_{S_{d}} S \Phi_{u}^{(2)} H_{d}
\end{aligned}
$$

The superpotential in Eq. (A.1) and Eq. (A.15) is the the most general one allowed by a $U(1)_{Z} \times Z_{3}$ symmetry with quantum numbers given in Table 5 , where $H_{u}$ is defined as that field that does not couple to $X$.

In order to get the minimal number of parameters, we impose both the DGS unification condition at the GUT scale

$\xi_{D}\left(M_{\mathrm{GUT}}\right)=\xi_{T}\left(M_{\mathrm{GUT}}\right)=\xi$.

and the Higgs-messenger mixing condition (for simplicity at the messenger scale)

$\lambda_{S_{d}}(M) y_{t}(M)=\lambda_{t}(M) \lambda(M)$.

The superpotential couplings leads to additional contributions to soft terms, on top of the soft terms from minimal GM in Eq. (A.3), from the DGS Model in Eqs. (A.6), (A.7), (A.9) and the U model in Eqs. (A.12), (A.13), (A.14). These new terms are the mixed contributions of the form

$$
\begin{aligned}
\Delta m_{H_{d}}^{2} & =3\left(\xi_{T}^{2} \lambda_{S_{d}}^{2}+\xi_{D}^{2} \lambda_{S_{d}}^{2}\right) \tilde{m}^{2}, \\
\Delta \tilde{m}_{S}^{2} & =\left(12 \xi_{T}^{2} \lambda_{S_{d}}^{2}+16 \xi_{D}^{2} \lambda_{S_{d}}^{2}+6 \xi_{D}^{2} \lambda_{t}^{2}\right) \tilde{m}^{2}, \\
\Delta \tilde{m}_{Q_{3}}^{2} & =\xi_{D}^{2} \lambda_{t}^{2} \tilde{m}^{2}, \\
\Delta \tilde{m}_{U_{3}}^{2} & =2 \xi_{D}^{2} \lambda_{t}^{2} \tilde{m}^{2} .
\end{aligned}
$$

\section{References}

1. J.L. Evans, M. Ibe, T.T. Yanagida, Phys. Lett. B 705, 342 (2011). https://doi.org/10.1016/j.physletb.2011.10.031

2. T. Jelinski, J. Pawelczyk, K. Turzynski, Phys. Lett. B 711, 307 (2012). https://doi.org/10.1016/j.physletb.2012.04.011 
3. J.L. Evans, M. Ibe, S. Shirai, T.T. Yanagida, Phys. Rev. D 85, 095004 (2012). https://doi.org/10.1103/PhysRevD.85.095004

4. Z. Kang, T. Li, T. Liu, C. Tong, J.M. Yang, Phys. Rev. D 86, 095020 (2012). https://doi.org/10.1103/PhysRevD.86.095020

5. N. Craig, S. Knapen, D. Shih, Y.Zhao, JHEP 03, 154 (2013). https:// doi.org/10.1007/JHEP03(2013)154

6. M. Abdullah, I. Galon, Y. Shadmi, Y. Shirman, JHEP 1306, 057 (2013). https://doi.org/10.1007/JHEP06(2013)057

7. H.D. Kim, D.Y. Mo, M.S. Seo, Eur. Phys. J. C 73(6), 2449 (2013). https://doi.org/10.1140/epjc/s10052-013-2449-z

8. P. Byakti, T.S. Ray, JHEP 1305, 055 (2013). https://doi.org/10. 1007/JHEP05(2013)055

9. N. Craig, S. Knapen, D. Shih, JHEP 08, 118 (2013). https://doi. org/10.1007/JHEP08(2013)118

10. J.A. Evans, D. Shih, JHEP 1308, 093 (2013). https://doi.org/10. 1007/JHEP08(2013)093

11. L. Calibbi, P. Paradisi, R. Ziegler, JHEP 1306, 052 (2013). https:// doi.org/10.1007/JHEP06(2013)052

12. T. Jelinski, JHEP 1309, 107 (2013). https://doi.org/10.1007/ JHEP09(2013) 107

13. I. Galon, G. Perez, Y. Shadmi, JHEP 1309, 117 (2013). https://doi. org/10.1007/JHEP09(2013)117

14. W. Fischler, W. Tangarife, JHEP 1405, 151 (2014). https://doi.org/ 10.1007/JHEP05(2014)151

15. S. Knapen, D. Shih, JHEP 1408, 136 (2014). https://doi.org/10. 1007/JHEP08(2014)136

16. R. Ding, T. Li, F. Staub, B. Zhu, JHEP 1403, 130 (2014). https:// doi.org/10.1007/JHEP03(2014)130

17. L. Calibbi, P. Paradisi, R. Ziegler, Eur. Phys. J. C 74(12), 3211 (2014). https://doi.org/10.1140/epjc/s10052-014-3211-x

18. A. Basirnia, D. Egana-Ugrinovic, S. Knapen, D. Shih, JHEP 06, 144 (2015). https://doi.org/10.1007/JHEP06(2015)144

19. T. Jelinski, PoS CORFU2014, 089 (2015)

20. T. Jelinski, J. Gluza, Phys. Lett. B 751, 541 (2015). https://doi.org/ 10.1016/j.physletb.2015.11.003

21. S. Knapen, D. Redigolo, D. Shih, JHEP 03, 046 (2016). https://doi. org/10.1007/JHEP03(2016)046

22. M. Badziak, M. Olechowski, S. Pokorski, JHEP 06, 043 (2013). https://doi.org/10.1007/JHEP06(2013)043

23. A. Delgado, G.F. Giudice, P. Slavich, Phys. Lett. B 653, 424 (2007). https://doi.org/10.1016/j.physletb.2007.07.057

24. B. Allanach, M. Badziak, C. Hugonie, R. Ziegler, Phys. Rev. D 92(1), 015006 (2015). https://doi.org/10.1103/PhysRevD.92. 015006
25. B.C. Allanach, M. Badziak, G. Cottin, N. Desai, C. Hugonie, R. Ziegler, Eur. Phys. J. C 76(9), 482 (2016). https://doi.org/10.1140/ epjc/s10052-016-4330-3

26. M. Aaboud et al., JHEP 01, 055 (2018). https://doi.org/10.1007/ JHEP01(2018)055

27. G.F. Giudice, R. Rattazzi, Nucl. Phys. B 511, 25 (1998). https:// doi.org/10.1016/S0550-3213(97)00647-0

28. R. Barbieri, G.F. Giudice, Nucl. Phys. B 306, 63 (1988). https:// doi.org/10.1016/0550-3213(88)90171-X

29. T. Gherghetta, B. von Harling, A.D. Medina, M.A. Schmidt, JHEP 02, 032 (2013). https://doi.org/10.1007/JHEP02(2013)032

30. K. Agashe, Y. Cui, R. Franceschini, JHEP 02, 031 (2013). https:// doi.org/10.1007/JHEP02(2013)031

31. S. Schael et al., Eur. Phys. J. C 47, 547 (2006). https://doi.org/10. 1140/epjc/s2006-02569-7

32. U. Ellwanger, J.F. Gunion, C. Hugonie, JHEP 02, 066 (2005). https://doi.org/10.1088/1126-6708/2005/02/066

33. U. Ellwanger, C. Hugonie, Comput. Phys. Commun. 175, 290 (2006). https://doi.org/10.1016/j.cpc.2006.04.004

34. D. Dercks, N. Desai, J.S. Kim, K. Rolbiecki, J. Tattersall, T. Weber, Comput. Phys. Commun. 221, 383 (2017). https://doi.org/10.1016/ j.cpc. 2017.08 .021

35. T. Sjöstrand, S. Ask, J.R. Christiansen, R. Corke, N. Desai, P. Ilten, S. Mrenna, S. Prestel, C.O. Rasmussen, P.Z. Skands, Comput. Phys. Commun. 191, 159 (2015). https://doi.org/10.1016/j.cpc.2015.01. 024

36. J. de Favereau, C. Delaere, P. Demin, A. Giammanco, V. Lemaître, A. Mertens, M. Selvaggi, JHEP 02, 057 (2014). https://doi.org/10. 1007/JHEP02(2014)057

37. M. Aaboud et al., JHEP 09, 084 (2017). https://doi.org/10.1007/ JHEP09(2017)084

38. M. Aaboud et al., Phys. Rev. D 97(11), 112001 (2018). https://doi. org/10.1103/PhysRevD.97.112001

39. G. Belanger, U. Ellwanger, J.F. Gunion, Y. Jiang, S. Kraml, J.H. Schwarz, JHEP 01, 069 (2013). https://doi.org/10.1007/ JHEP01(2013)069

40. CMS Collaboration, CMS-PAS-HIG-17-0137 (2017)

41. A.M. Sirunyan et al., JHEP 03, 166 (2018). https://doi.org/10.1007/ JHEP03(2018)166 